\title{
Novel Germline RUNX1 Mutation Associated with Familial Thrombocytopenia as well as B-Acute Lymphoblastic Leukemia: A Case Report and Review of the Literature
}

\author{
Nabin Karki ${ }^{a} \quad$ Natasha Savage ${ }^{b}$ Abdullah Kutlar ${ }^{a}$ \\ aDivision of Hematology/Oncology, Augusta University, Augusta, GA, USA; ${ }^{b}$ Department of \\ Pathology, Augusta University, Augusta, GA, USA
}

Keywords

RUNX1 · Familial platelet disorder · Acute lymphoblastic leukemia $\cdot$ B-ALL

\begin{abstract}
Germline RUNX1 mutations lead to a rare form of autosomal-dominant familial thrombocytopenia with a predisposition for myeloid malignancies and are classified as distinct entities by the WHO. We report a case of B lymphoblastic leukemia developing in a patient with a familial RUNX1 mutation, which is a first in the literature. An FLT3-ITD mutation as well as a balanced chromosomal translocation $t(1 ; 7)$ was present at the time of diagnosis of leukemia, favoring the theory that additional hits or mutations are necessary for malignant transformation in patients with a germline $R U N X 1$ mutation. The transformed disease runs an aggressive course compared to the same malignancy associated with a somatic RUNX1 mutation. Additionally, family members should be screened for the mutation, followed up clinically if they carry the mutation, and should not be used as stem cell donors to treat the affected relatives.
\end{abstract}

(C) 2021 The Author(s).

Published by S. Karger AG, Basel

\section{Introduction}

RUNX1 (runt-related transcription factor 1 ) is a master regular of hematopoiesis and is transcribed by the RUNX1 gene, located in chromosome 21q. Germline mutations in RUNX1 cause autosomal-dominant familial platelet disorder (FPD) with a disposition to myeloid malignancies like myelodysplastic syndrome (MDS), acute myeloid leukemia (AML), chronic myelomonocytic leukemia (CMML), and rarely T lymphoblastic leukemia (T-ALL). These mutations are often unique to each family, with about 70 families described so far. FPD mani- 


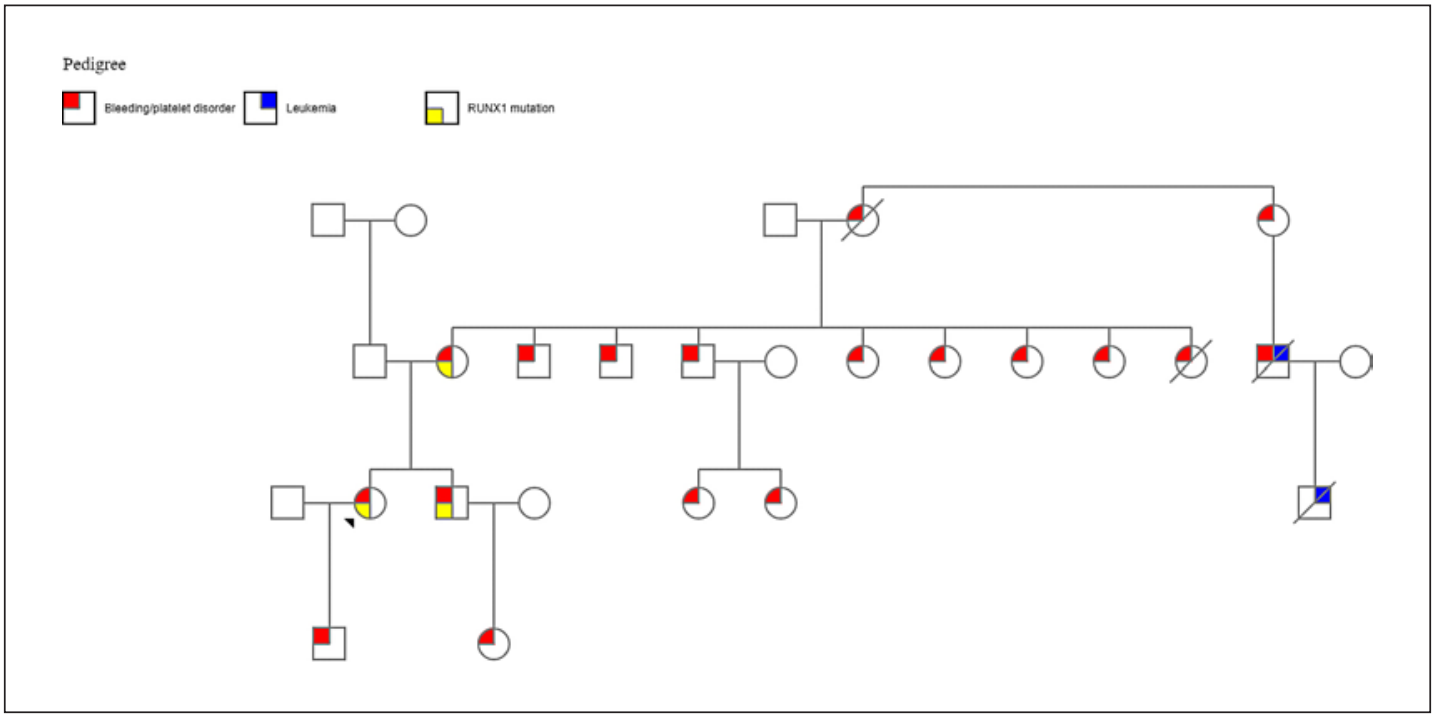

Fig. 1. Family pedigree: the arrowhead indicates the patient.

fests as mild-moderate thrombocytopenia with normal-sized platelets and functional platelet defects leading to prolonged bleeding. Approximately half of affected individuals develop hematologic malignancies in adulthood, mostly of myeloid origin. A few cases of T-ALL and 1 case of childhood B-ALL have been described so far in the literature in association with a germline RUNX1 mutation. We describe a novel RUNX1 mutation in an adult female with FPD evolving into B-ALL [1].

\section{Case Presentation}

A 44-year-old Caucasian female was seen due to a long history excessive bleeding. The patient had significant bleeding at menarche refractory to combined oral contraceptive pills, vaginal delivery at the age of 22 years with prolonged bleeding, menometrorrhagia requiring hysterectomy aged 27 years, and excessive bleeding with appendectomy and wisdom teeth removal. She had been treated with platelet transfusions and desmopressin. She reported increased bruising but denied bleeding from the gastrointestinal tract, gums, and epistaxis.

There were multiple family members across three generations of either sex with similar bleeding issues, all of whom were on her maternal side. The bleeding phenotype ranged from milder bleeding issues to fatal bleeding complications. The patient's mother had a male cousin with "adult-onset leukemia," and his son had died of "childhood leukemia" at the age of 10 years as well. The full pedigree is shown below (Fig. 1).

The laboratory findings showed a low normal platelet number (approx. 150,000/ $\mu \mathrm{L}$ ). The white cell and red cell lineage including morphology was unremarkable without any dysplastic forms. Platelet functional studies showed an abnormal platelet function assay (PFA). PFA collagen/epinephrine was >300 s (normal: 109-183 s), but PFA collagen/ adenosine diphosphate (ADP) was $100 \mathrm{~s}$ (normal: 75-110 s). A decreased secondary response to ADP/collagen was noted on platelet aggregation studies. A normal response was seen to ristocetin. The von Willebrand factor antigen level was normal. Flow cytometry of platelet glycoprotein expression revealed a normal expression of GP1b and GPIIb/IIIa, ruling out Bernard-Soulier and Glanzmann thrombasthenia. 


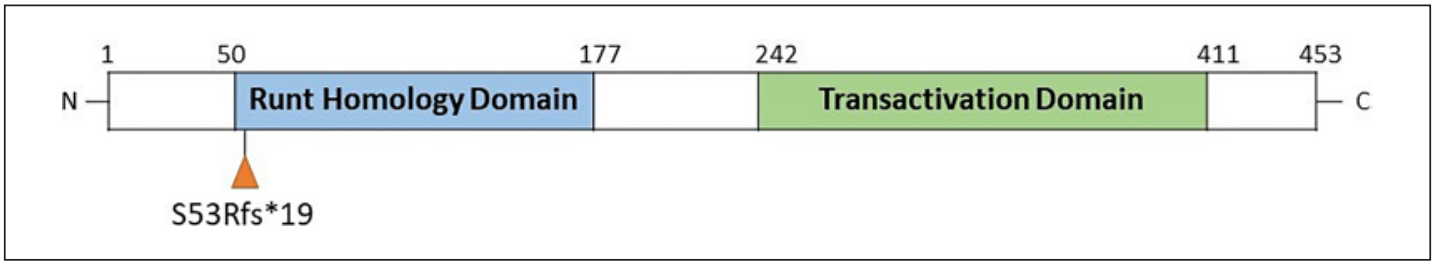

Fig. 2. Frameshift mutation in RHD: c.159delC causing p.Ser53ArgfsTer19.

The patient's brother was found to have a c159del (S53Rfs*9) RUNX1 mutation (frameshift in RUNX1). This RUNX1 mutation (c159 deletion) was confirmed in several family members, including the patient (Fig. 2). Based on the variant allele frequency of $47.38 \%$, it was a heterozygous variant. This finding established the diagnosis of FPD related to the RUNX1 mutation. A year later, the patient developed fatigue, headaches, and palpitations with minimal activity and was found to have profound pancytopenia. A bone marrow biopsy showed $70 \%$ blasts (Fig. 3 ).

The blasts were positive for TdT (variable), CD34 (strong, granular cytoplasmic), CD20 (20\% blasts), CD79a (20\% blasts), PAX5 (variable), and CD19 (dim). They were negative for MPO, CD68, other monocytic markers, factor VIII, spectrin, E-cadherin, CD117, cCD3, sCD3, and CD10. Although a strong expression of CD19 and CD10 were not noted, the overall features were most consistent with B-ALL.

An FLT3-ITD mutation was detected, and karyotyping revealed 46,XX,der(7)t(1;7) (q12; q31)[9]/46,XX[11]. Her cerebrospinal fluid was negative for leukemic involvement. The patient was started on a hyperCVAD protocol and received a hyperCVAD cycle $1 \mathrm{~A}$ as an inpatient. She received intrathecal methotrexate and intrathecal cytarabine for spinal prophylaxis. On day 14, a bone marrow biopsy showed morphologic remission with normal cytogenetic/FISH studies. The patient received the next cycle (1B) of hyperCVAD and was evaluated for an allogenic stem cell transplant. Subsequently, she underwent a matched unrelated donor allogenic stem cell transplant with $5.39 \times 10^{6} \mathrm{CD} 34$ cells $/ \mathrm{kg} 3$ months after the diagnosis of leukemia. Myeloablative conditioning with busulfan, cyclophosphamide, and antithymocyte globulin was utilized. Engraftment was achieved 2 weeks (day 15) after stem cell infusion. On post-transplant day 100, a bone marrow biopsy confirmed morphologic remission with no evidence of minimal residual disease. The treatment course was complicated by mucositis, neutropenic fever, infection of sinuses and scalp, gastrointestinal graft versus host disease (GVHD), and platelet refractoriness.

Sirolimus was used for GVHD prophylaxis and was stopped after 3 months due to thrombotic microangiopathy. Five months after her allogenic stem cell transplant, the patient developed deep venous thrombosis of the left lower extremity, which was successfully treated with low-molecular-weight heparin. Nine months after the stem cell transplant, the patient was profoundly neutropenic and presented with right arm cellulitis and right submandibular sialadenitis. A bone marrow biopsy was without morphologic evidence of leukemia, but was significant for hypocellularity with reduced erythropoiesis and granulopoiesis. The patient was thought to have developed aplastic anemia. Chimerism studies showed $100 \%$ donor myeloid cells, $96 \%$ donor T cells, and 100\% donor B cells. Unfortunately, the patient succumbed todeathfromneutropenicsepsisshortlythereafter, whilewaitingto receive eltrombopag or romiplostim. 


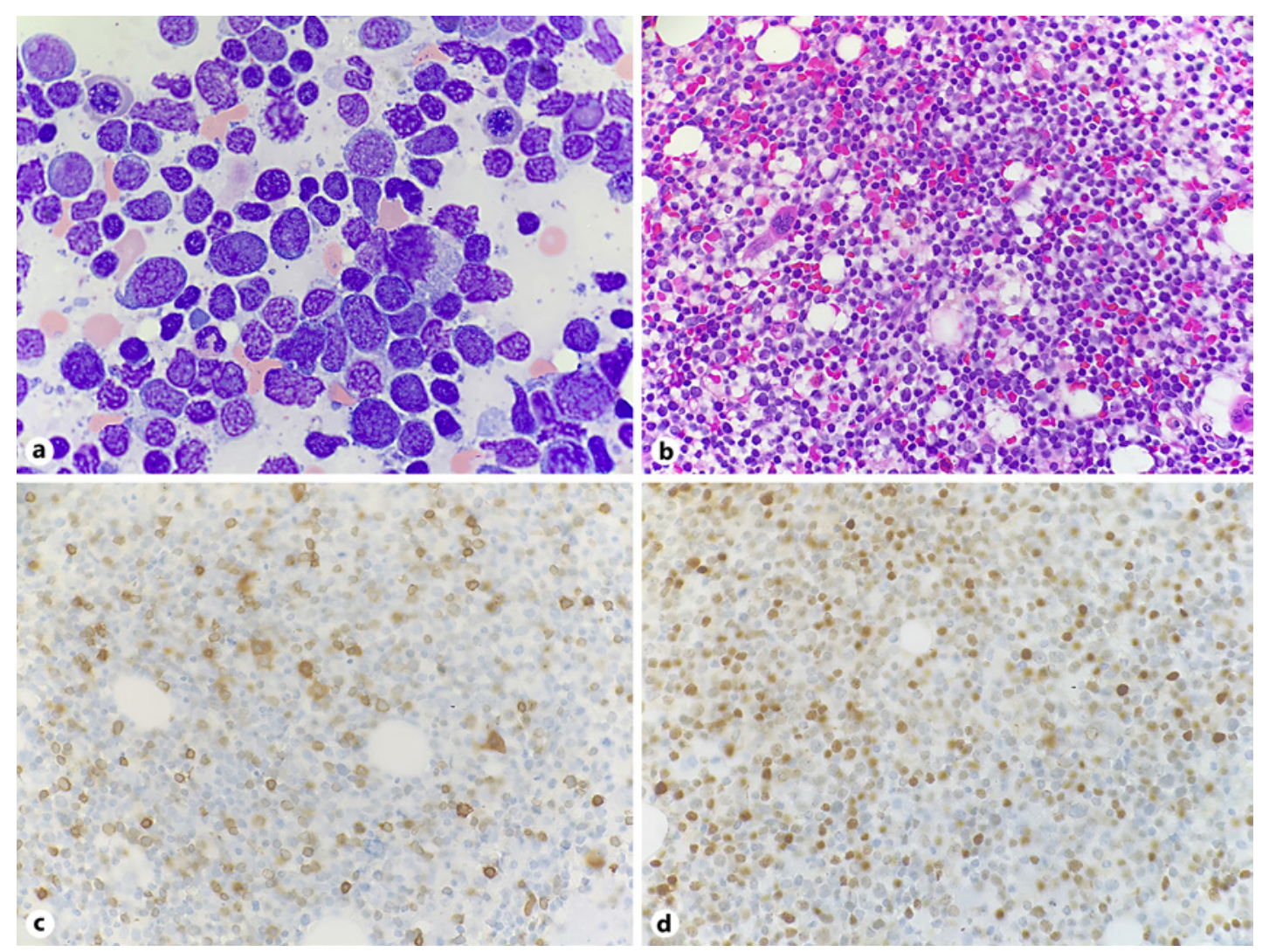

Fig. 3. a Morphologic examination of the bone marrow aspirate revealed decreased trilineage hematopoiesis. The majority of the cellularity consisted of blasts which were medium in size with increased nuclear to cytoplasmic ratios, a more open chromatin pattern, and mostly inconspicuous nucleoli. A subset of blasts had a "hand mirror" morphology. Lymphoglandular bodies were present in the background. Wright-Giemsa stain. Original magnification, $\times 1,000$. b Morphologic examination of the bone marrow biopsy revealed a hypercellular marrow with decreased trilineage hematopoiesis including few scattered megakaryocytes. The majority of the cellularity consisted of immature mononuclear cells, consistent with blasts. The blasts were medium in size with increased nuclear to cytoplasmic ratios and an open chromatin pattern. Hematoxylineosin stain. Original magnification, $\times 500$. c Morphologic examination of CD79a immunohistochemical stain performed on the bone marrow biopsy revealed a subset of blasts with a cytoplasmic expression. A few plasma cells were also noted with positive staining. These plasma cells stain darker than the blasts and have a more abundant, eccentric cytoplasm. Original magnification, $\times 500$. d Morphologic examination of PAX5 immunohistochemical stain performed on the bone marrow biopsy revealed a significant subset of blasts with nuclear expression. Original magnification, $\times 500$.

\section{Discussion}

RUNX1 (also known as AML1, CBFA2, or PEPB2A) is a critical transcription factor for definite embryonic hematopoiesis [2]. RUNX1 genes encode a DNA-binding subunit that contains a highly conserved runt-homology domain (RHD) for sequence-specific DNA binding. The RUNX1 protein complexes with core-binding factor to form a heterodimeric core-binding transcription factor that regulates many genes important in hematopoiesis. More than 40 mutations have been described so far, mostly missense or deletion [3]. Most of the mutations causing FPD/AML are located in RHD, leading to disruption of DNA binding ability. Heterozygous germline mutations in the RUNX1 gene lead to FPD with propensity to myeloid malignancies [4]. 
Karki et al.: Novel Germline RUNX1 Mutation

Table 1. Reported cases of germline RUNX1 transformation to acute lymphoblastic leukemia

\begin{tabular}{|c|c|c|c|c|c|c|c|}
\hline Study & $\begin{array}{l}\text { Age, } \\
\text { years }\end{array}$ & Sex & ALL type & Mutation type & Additional hit(s) & $\begin{array}{l}\text { Complete } \\
\text { remission after } \\
\text { induction } \\
\text { chemotherapy }\end{array}$ & $\begin{array}{l}\text { Post-remission } \\
\text { course }\end{array}$ \\
\hline Nishimoto et al. [13] & 20 & Male & T-ALL & R174X (non-sense) & $\mathrm{t}(1 ; 7)$ & Yes & $\begin{array}{l}\text { Allogenic stem cell } \\
\text { transplant from } \\
\text { unrelated donor }\end{array}$ \\
\hline Manchev et al. [14] & 42 & Male & T-ALL & R174Q (missense) & TET2 & $\begin{array}{l}\text { Yes (required } 2 \\
\text { induction } \\
\text { courses) }\end{array}$ & $\begin{array}{l}\text { Developed AML } \\
\text { later }\end{array}$ \\
\hline Prebet et al. [15] & 42 & Male & T-ALL & R174Q (missense) & ASLX1 & $\begin{array}{l}\text { Yes (required } 2 \\
\text { induction } \\
\text { courses) }\end{array}$ & Refused transplant \\
\hline Linden et al. [16] $]^{a}$ & 3 & Female & B-ALL & c.568G >A (missense) & $\begin{array}{l}\text { Trisomy } 12 \mathrm{p}, \\
\text { trisomy/ tetrasomy } \\
21 \mathrm{q}\end{array}$ & Yes & Alive after 2 years \\
\hline This study & 47 & Female & B-ALL & c.159del (frameshift) & FLT3-ITD, t(1;7) & Yes & $\begin{array}{l}\text { Developed aplastic } \\
\text { anemia after } \\
\text { allogenic stem cell } \\
\text { transplant }\end{array}$ \\
\hline
\end{tabular}

\footnotetext{
${ }^{\text {a }}$ Linden et al. described childhood B-ALL and thrombocytopenia due to RUNX1 mutation in a 3-year-old girl; however, the authors were not able to prove the germline nature of the mutation.
}

FPD was first described in 1969, and the first family with FPD with a propensity to myeloid malignancies was reported in 1978 [5, 6]. The chromosome loci for RUNX1, 21q, was identified in 1996 by linkage analysis [7]. Haploinsufficiency of RUNX1 due to nonsense or missense mutations of one allele was shown to be responsible for FPD with a propensity to myeloid malignancies in a landmark study published in Nature [4]. RUNX1 is expressed in hematopoietic stem cells and differentiating myeloid and lymphoid cells [8]. Haploinsufficiency of RUNX1 might constitutively activate MYH10 expression and affect megakaryocyte polyploidization [9]. A possible other theory is that downregulation of MYL9 due to RUNX1 haploinsufficiency could affect abnormal platelet production and function [10]. Additional events are necessary in malignant transformation, such as FLT3-ITD [11].

Somatic mutations in RUNX1 are common in myeloid malignancies [2]. RUNX1 translocations are among the more frequent chromosomal rearrangements in human leukemia, most often seen as $t(8 ; 21)$. However, they are distinct from germline mutations and distinct from FPD with a propensity to myeloid malignancies. FPD with a propensity to myeloid malignancies is inherited in an autosomal-dominant fashion with a variable expression. Platelet dysfunction is almost universal with both quantitative and qualitative disorders, mostly mild to moderate. The average age at diagnosis is 34.16 years [11]. Approximately $20-60 \%$ of affected individuals develop myeloid malignancies, such as AML, MDS, or CMML [12]. An additional genetic event is thought to be necessary for transformation to leukemia. Three cases of T-ALL and 1 case of childhood B-ALL have been described, usually with a discernible second hit [13-16] (Table 1).

Our case represents the first confirmed B-ALL in an adult patient with germline RUNX1 mutation. The patient had c159del (S53Rfs*9) (frameshift mutation in RUNX1) which has been reported as a pathogenic variant but not described in association with FPD or AML [17]. The frameshift starts in the runt domain in exon 3, like most other RUNX1 mutations known 
to be associated with FPD with a propensity to myeloid malignancies. This mutation is unique and was present in multiple family members. An association between the type of RUNX1 mutation, nature of secondary mutations, or type of leukemia has not been studied. FLT3-ITD mutations are common in AML, and $t(1 ; 7)$ has been seen in T-ALL, in contrast to our case, which had features most compatible with B-ALL. However, it is likely that the FLT3-ITD mutation and $t(1 ; 7)$ represent additional hits that predisposed FPD to acute leukemia. Importantly, family members should be screened for this mutation prior to consideration for stem cell donation for allogenic transplantation. It seems prudent that carriers of germline RUNX1 mutation be followed more closely.

Although core-binding leukemia comprise a favorable class of leukemia, the outcomes are poor for FPD with a propensity to myeloid malignancies when compared to those with a somatic RUNX1 mutation. Allogenic stem cell transplantation is pursued for eligible patients. However, it is important to screen family members for RUNX1 mutations, and RUNX1-positive family members should not be used. Our patient developed aplastic anemia after successful matched unrelated donor allogenic stem cell transplant and succumbed to an infectious complication while retaining excellent donor chimerism. This course is not typical of RUNX1 germline status and remains unexplained.

\section{Conclusion}

FPD with propensity to myeloid malignancies is caused by inherited mutations in the RUNX1 gene. Thrombocytopenia is a common finding; clinicians must monitor these patients closely for the development of hematologic malignances. About half will develop hematologic malignancies aged in their 30s or 40s. Although myeloid malignancies are commonly known to occur, lymphoid malignancies are now also being reported. Here, we described the first confirmed B-ALL and a novel RUNX1 germline mutation in an adult.

\section{Acknowledgements}

We are grateful to Sally S. Agersborg, MD, PhD (NeoGenomics Laboratories Inc. Aliso Viejo, CA, USA), Alison E. Spellman, MD (Summit Cancer Care, Savannah, GA, USA), and Mara Banks, MD (University of Utah, Salt Lake City, UT, USA).

\section{Statement of Ethics}

Written informed consent was obtained for the publication of this case report from the patient's next of kin.

\section{Conflict of Interest Statement}

The authors have no conflicts of interest to declare.

\section{Funding Sources}

There was no funding in relation to this work.

\section{Karger'}




\section{Case Reports in Oncology}

\begin{tabular}{l|l}
\hline Case Rep Oncol 2021;14:439-445 \\
\hline DOI: 10.1159/000512016 & $\begin{array}{l}\text { @ 2021 The Author(s). Published by S. Karger AG, Basel } \\
\text { www.karger.com/cro }\end{array}$ \\
\hline
\end{tabular}

Karki et al.: Novel Germline RUNX1 Mutation

\section{Author Contributions}

N.K. wrote the case report and performed the literature search. A.K. treated the patient. A.K. and N.S. reviewed and edited the manuscript. N.S. performed and interpreted all the bone marrow studies.

\section{References}

1 College of American Pathologists. Abstracts and case studies from the College of American Pathologists 2018 annual meeting (CAP18). Arch Pathol Lab Med. 2018 Sep 1;142(9):e2-202. Available from:https://doi.org/ 10.5858/arpa.2018-0293-AB.

2 Growney JD, Shigematsu H, Li Z, Lee BH, Adelsperger J, Rowan R, et al. Loss of Runx1 perturbs adult hematopoiesis and is associated with a myeloproliferative phenotype. Blood. 2005 Jul;106(2):494-504.

3 Morgan NV, Daly ME. Gene of the issue: RUNX1 mutations and inherited bleeding. Platelets. 2017 Mar;28(2): 208-10.

4 Song WJ, Sullivan MG, Legare RD, Hutchings S, Tan X, Kufrin D, et al. Haploinsufficiency of CBFA2 causes familial thrombocytopenia with propensity to develop acute myelogenous leukaemia. Nat Genet. 1999 Oct; 23(2):166-75.

5 Weiss HJ, Chervenick PA, Zalusky R, Factor A. A familial defect in platelet function associated with imapired release of adenosine diphosphate. N Engl J Med. 1969 Dec;281(23):1264-70.

6 Luddy RE, Champion LA, Schwartz AD. A fatal myeloproliferative syndrome in a family with thrombocytopenia and platelet dysfunction. Cancer. 1978 May;41(5):1959-63.

7 Ho CY, Otterud B, Legare RD, Varvil T, Saxena R, DeHart DB, et al. Linkage of a familial platelet disorder with a propensity to develop myeloid malignancies to human chromosome 21q22.1-22.2. Blood. 1996 Jun;87(12): 5218-24.

8 North TE, Stacy T, Matheny CJ, Speck NA, de Bruijn MF. Runx1 is expressed in adult mouse hematopoietic stem cells and differentiating myeloid and lymphoid cells, but not in maturing erythroid cells. Stem Cells. 2004; 22(2):158-68.

9 Lordier L, Bluteau D, Jalil A, Legrand C, Pan J, Rameau P, et al. RUNX1-induced silencing of non-muscle myosin heavy chain IIB contributes to megakaryocyte polyploidization. Nat Commun. 2012 Mar;3:717.

10 Jalagadugula G, Mao G, Kaur G, Goldfinger LE, Dhanasekaran DN, Rao AK. Regulation of platelet myosin light chain (MYL9) by RUNX1: implications for thrombocytopenia and platelet dysfunction in RUNX1 haplodeficiency. Blood. 2010 Dec;116(26):6037-45.

11 Hayashi Y, Harada Y, Huang G, Harada H. Myeloid neoplasms with germ line RUNX1 mutation. Int J Hematol. 2017 Aug;106(2):183-8.

12 Ganly P, Walker LC, Morris CM. Familial mutations of the transcription factor RUNX1 (AML1, CBFA2) predispose to acute myeloid leukemia. Leuk Lymphoma. 2004 Jan;45(1):1-10.

13 Nishimoto N, Imai Y, Ueda K, Nakagawa M, Shinohara A, Ichikawa M, et al. T cell acute lymphoblastic leukemia arising from familial platelet disorder. Int J Hematol. 2010 Jul;92(1):194-7.

14 Manchev VT, Bouzid H, Antony-Debré I, Leite B, Meurice G, Droin N, et al. Acquired TET2 mutation in one patient with familial platelet disorder with predisposition to AML led to the development of pre-leukaemic clone resulting in T2-ALL and AML-M0. J Cell Mol Med. 2017 Jun;21(6):1237-42.

15 Prebet T, Carbuccia N, Raslova H, Favier R, Rey J, Arnoulet C, et al. Concomitant germ-line RUNX1 and acquired ASXL1 mutations in a T-cell acute lymphoblastic leukemia. Eur J. Haematol. 2013;91(3):277-9.

16 Linden T, Schnittger S, Groll AH, Juergens H, Rossig C. Childhood B-cell precursor acute lymphoblastic leukaemia in a patient with familial thrombocytopenia and RUNX1 mutation. Br J Haematol. 2010;151(5): 528-30.

17 National Center for Biotechnology Information. ClinVar (VCV000561228.1) [Internet]. Cited 2020 Jun 6. Available from: https://www.ncbi.nlm.nih.gov/clinvar/variation/VCV000561228.1. 